loads, providing the ratio of breakdown to locked-rotor torque is correct. However, standardizing on increased values for these torques would raise the motor weights as well as that of associated equipment for applications not requiring a reserve for peak loads or starting. The ratio of breakdown to locked-rotor torque, although not ideal for all applications, is nearly correct for the majority of them.

\section{Illustrations and Characteristic Curves}

Figure 1 shows a three-quarter-horsepower continuous 400-cycle 200-volt three-phase motor designed to operate in accordance with the requirements previously outlined. The weight of this motor is 3.4 pounds. It is about 40 per cent lighter than a d-c motor having the same horsepower and speed rating. Other 400-cycle and 24-volt d-c motors with corresponding ratings have about the same ratio of weight.

Figure 2 shows characteristic curves plotted from test data taken on the threequarter-horsepower motor at normal voltage, frequency, altitude, and ambient temperature. Speeds, efficiencies, power factors, amperes, and watts output which are plotted against torques are included.

Figure 3 illustrates schematically the construction used for continuous-rated motors with outputs from one eighth to 25 horsepower. That used for intermittent-rated motors is similar except the ventilation ducts, overhung fans, and fan covers are omitted.

Major design features used in these motors are summarized as follows:

1. Magnetic structures fabricated from high-grade silicon steel with special anneal to permit high flux, good permeability, and low iron loss.

2. Insulation. Class- $B$ material for insulating slots and for between-phase protection. Generally the stator windings are of heavy Formex-glass-covered wire to provide greater protection against heat shock. Stator core complete with windings impregnated with special moisture-resisting varnish.

3. Squirrel-cage-type rotors with brazed copper or brass windings. Protected with anticorrosion treatment.

4. Through ventilation in continuous-rated motors for minimum composition and weight. Double end-turn ventilation in intermittent-rated motors.

5. Shells made by expanding thin steel tubing to obtain light weight, rigid construction, and a temperature coefficient the same as that of the stator.

6. Magnesium or aluminum end shields for light weight.

7. Heat-treated alloy steel shafts for strength and dependability. Hardened shaft extensions with or without pinions or splines.

\title{
Analysis and Design of
Selsyn System
}

\author{
JOSEPH MANILDI \\ MEMBER AIEE
}

Synopsis: At tfre present time there are several remote-indicating systems in common usage in industry. These types include the conventional a-c Selsyn, a-c Magnesyn system, and the d-c Selsyn. The d-c Selsyn embodies two features which present a distinct advantage in aircraft systems.

1. The system does not require an a-c source of power.

2. The system requires only three wires between transmitter and receiver, whereas all other types of systems require at least five wires leading from the transmitter to the receiver.

To offset somewhat these advantages, all d-c Selsyn systems now in operation have a very slight natural error which, although not important in some applications, becomes very undesirable in applications where a high degree of accuracy is required. In this paper the author presents a rigorous analysis of a conventional d-c Selsyn remote-indicating system. The first part of the analysis shows that a natural error exists in the system, which has a 60-degree cycle and which reaches a maximum value of $1.1 \mathrm{de}$ grees. The error is zero at each 30 -degree point and reaches a maximum almost midway between the 30 -degree points. The natural error of the Selsyn system arises from the fact that the transmitter resistance is a linear function of the transmitter angle $\theta$. This natural error may be overcome by designing a transmitter resistance in a nonlinear manner. The equation is developed for the necessary variation of the transmitter resistance with the transmitter angle. Furthermore, the shape of the toroid on which this resistor should be wound to give this desired variation, is derived therefrom. The paper further presents optimum values of resistances for both transmitter and receiver, in order that the greatest amount of

Paper 45-101, recommended by the AIEE committee on instruments and measurements for publication in AIEE Transactions. Manuscript submitted February 3, 1945; made available for printing March 21, 1945.

Joseph ManiLd is on the staff of the department of electrical engineering, California Institute of Technology, Pasadena, Calif.

8. Double-shield-type permanently lubricated ball bearings housed in a steel insert in the end-shield castings to assure long life. 9. Protective finishes. Magnesium or aluminum castings treated against corrosion and primed with zinc chromate. Steel parts other than stator and rotor punchings cadmium- or zinc-plated. External parts except mounting surface-have black wrinkle finish.

\section{Conclusions}

Present trends indicate an increasing demand for 400-cycle electric systems for torque may be transmitted to the receiver for a given amount of power.

\section{Determination of Natural Error in a Conventional D-C Selsyn System}

$\mathbf{T}$ HE INVESTIGATION of the d-c Selsyn embodies two separate questions.

1. If no frictional error is assumed, what are the magnitudes and characteristics of natural errors which may exist in the instrument, and how may they be corrected?

2. For a given amount of power consumption how should the instrument be constructed to give best follower properties from transmitter to receiver; that is, for a given amount of friction, how can the frictional error be reduced to a minimum?

In analyzing the first question, we consider the conventional d-c Selsyn shown in Figure 1. The receiver coils $A B, B C$, and $C A$ are wound with their magnetic axes at 120 degrees to each other and connected in a delta connection. Points $A$, $B$, and $C$ are connected to points $A^{\prime}, B^{\prime}$, $C^{\prime}$, respectively, located at 120 -degree intervals on the transmitter winding. The battery contacts $D^{\prime}$ and $E^{\prime}$ are on a diameter and may be moved at will.

Ideally, the direction of the resultant field in the receiver should move in exact coincidence with the angle $\theta$ in the transmitter. There is no a priori reason for supposing that this situation will exist. Actually, as we shall see, if the resistances in the transmitter are linear with the angle $\theta$, the ideal situation does not exist: The amount of natural error introduced is determined in the following analysis:

We obtain first the current distribution in coils $A B, B C$, and $C A$ when the contacts $D^{\prime}$ and $E^{\prime}$ are rotated $\theta$ degrees from $A^{\prime}$ as shown. $0 \leqq \theta \leqq 60$ degrees. The transmitter is assumed to have a re-

both military and commercial airplanes. These trends are justified in part by the inherently light-weight rugged and dependable constructions possible in 400 cycle motors.

The information contained in the paper is that which seems most important. Many additional data have not been mentioned; however, it is hoped that the disclosures herein will assist in creating a better understanding of the motors and the performance to be expected from them. 
sistance of $R_{1}$ ohms per degree, or $120 R_{1}$ ohms per leg. The receiver is assumed to have a resistance of $120 R_{2} \mathrm{ohms}$ per leg. Each section of resistance in the circuit is numbered as shown in Figure 1, and the equivalent circuit is shown in Figure 2, with corresponding numbers and the value of each resistance shown. The points $C, A$, and $B$ are marked, since we are interested in the voltages between these points.

To simplify the analysis we replace the delta sections $3-4-7$ and $1-2-6$ by their equivalent wye sections.

The transformation relationships which replace a delta system $R_{1}-R_{2}-R_{3}$ by a wye system $r_{1}-r_{2}-r_{3}$ are given by

$$
\left.\begin{array}{l}
r_{1}=\frac{R_{2} R_{3}}{R_{1}+R_{2}+R_{3}} \\
r_{2}=\frac{R_{1} R_{3}}{R_{1}+R_{2}+R_{3}} \\
r_{3}=\frac{R_{1} R_{2}}{R_{1}+R_{2}+R_{3}}
\end{array}\right\}
$$

The foregoing relationships leave the current and voltages at the three terminals unchanged. after the transformation is made and the resistors 5 and 8 are combined. Values are shown. The resistance of the two parallel branches in Figure 3 are equal; hence half the total current flows in each branch. The system is further reduced in Figure 4, and we obtain for the total current

$$
2 i=\frac{60 E\left(R_{1}+R_{2}\right)}{5,400 R_{1} R_{2}+R_{1}{ }^{2}\left(-\theta^{2}+60 \theta+1,800\right)}
$$

The current in each branch is then

$$
i=\frac{30 E\left(R_{1}+R_{2}\right)}{5,400 R_{1} R_{2}+R_{\mathrm{h}}{ }^{2}\left(-\theta^{2}+60 \theta+1,800\right)}
$$

Figure 3 shows the equivalent circuit

Referring to Figure 3, we have the following voltages:

$$
\begin{aligned}
E_{C A} & =i\left(\frac{120 R_{1} R_{2}}{R_{1}+R_{2}}\right) \\
& =\frac{3,600 E r}{\left(-\theta^{2}+60 \theta+1,800\right)+5,400 r} \\
E_{A B} & =-i\left(\frac{R_{1} R_{2}(120-\theta)}{R_{1}+R_{2}}-\frac{\theta R_{1} R_{2}}{R_{1}+R_{2}}\right) \\
& =\frac{-30 E r(120-2 \theta)}{\left(-\theta^{2}+60 \theta+1,800\right)+5,400 r} \\
E_{B C} & =-i\left(\frac{R_{1} R_{2}(60+\theta)}{R_{1}+R_{2}}-\frac{R_{1} R_{2}(60-\theta)}{R_{1}+R_{2}}\right) \\
& =-\frac{60 E r \theta}{\left(-\theta^{2}+60 \theta+1,800\right)+5,400 r}
\end{aligned}
$$

where $r=R_{2} / R_{1}$. The currents in the coils are then, from Figure 1 ,

$$
\begin{gathered}
I_{C A}=-\frac{30 E}{R_{1}}\left[\frac{1}{\left(-\theta^{2}+60 \theta+1,800\right)+5,400 r}\right] \\
I_{A B}=-\frac{E}{2 R_{1}}\left[\frac{60-\theta}{\left(-\theta^{2}+60 \theta+1,800\right)+5,400 r}\right] \\
I_{B C}=-\frac{E}{2 R_{1}}\left[\frac{\theta \phi_{A B}}{\left(-\theta^{2}+60 \theta+1,800\right)+5,400 r}\right] \\
=K \phi_{B C}
\end{gathered}
$$

For a fixed number of turns, the magnetic fields due to each of the coils are proportional to the currents. Figure 5 shows the relative orientation of these fields when the current in each branch is positive. We denote the direction of the resultant field, $\phi$, with the $x$ axis by the angle $\theta^{\prime}$. When $\theta=0$ we see from equa tions 7,8 , and 9 that $\phi_{C A}$ and $\phi_{A B}$ are equal in magnitude, and $\phi_{B C}=0 . \phi_{C A}$ is positive, and $\phi_{A B}$ is negative. Hence when $\theta=0, \theta^{\prime}=0$.

To obtain the value of $\theta^{\prime}$ for any value
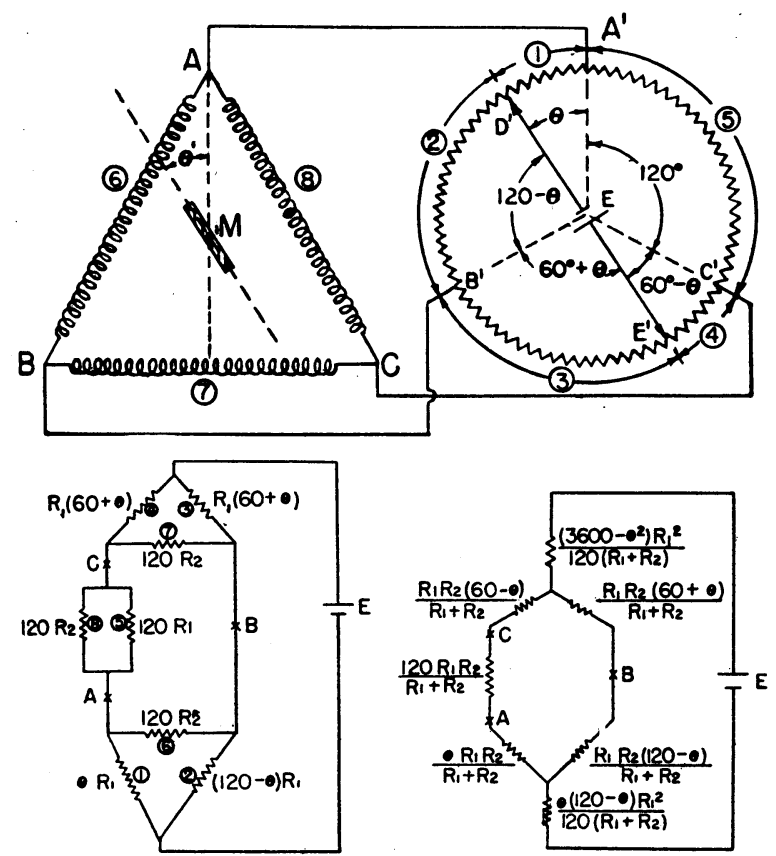

Figure 1 (left). D-c Selsyn transmitter and receiver

Figure 2 (lower left). Electric circuit of Figure 1

Figure 3 (center). Reduction of circuit in Figure 2

Figure 4 (below). Further reduction of circuit in Figure 2

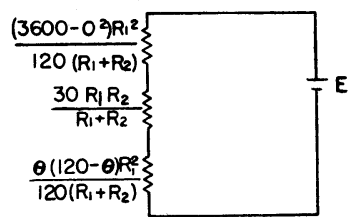

of $\theta$, we denote the $x$ and $y$ components of the resultant flux by $\phi_{x}$ and $\phi_{\nu}$. From Figure 5 we see that

$\phi_{x}=\frac{\sqrt{ } 3}{2}\left(\phi_{C A}-\phi_{A B}\right)$

$\phi_{y}=-\phi_{B C}+1 / 2\left(\phi_{C A}+\phi_{A B}\right)$

and

$\tan \theta^{\prime}=\frac{\phi_{y}}{\phi_{x}}$

Using equations 7,8 , and 9 and noting that the proportionality constant between flux and current is the same in every case, in conjunction with equations 10,11 , and 12 , we obtain

$\tan \theta^{\prime}=\sqrt{3} \frac{\theta}{120-\theta}$

It is important to note that equation 13 , which shows how the receiver angle $\theta^{\prime}$ varies with the transmitter angle $\theta$, is independent of the values of the resistances used in the Selsyn. Hence changing the values of the resistances can in no way compensate for natural error in the instrument.

Figure 6 shows a plot of $\theta^{\prime}$ versus $\theta$ for 60 degrees. Conditions repeat themselves every 60 degrees, of course, since at 60-degree intervals one battery contact is in coincidence- with a coil terminal. We see from the figure that the receiver gives an exact indication of the transmitter every 30 degrees. On one side of this point the receiver lags and on the other side it leads the transmitter.

If we denote by $\beta$ the difference of the receiver angle and the transmitter angle, we have

$\beta=\theta-\theta^{\prime}=\theta-\tan ^{-1}\left(\frac{\theta \sqrt{3}}{120-\theta}\right)$

It readily may be shown that this error angle is symmetrical in magnitude about the point $\theta=30$ degrees. Figure 7 shows a plot of $\beta$ versus $\theta$ for $0 \leqq \theta \leqq 60$ degrees.

By differentiating equation 14 with respect to $\theta$ and setting the result equal

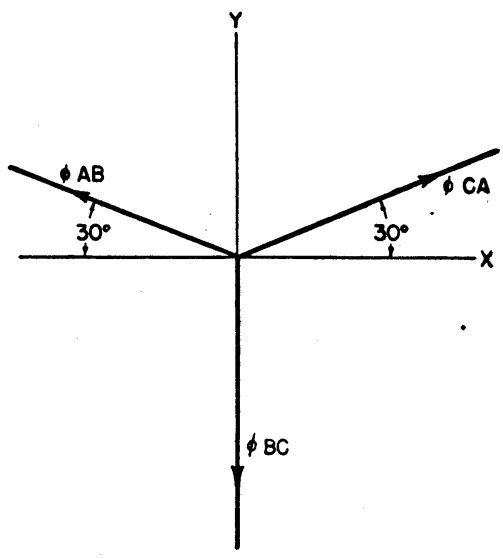

Figure 5. Space relationships of fluxes produced by receiver coils 


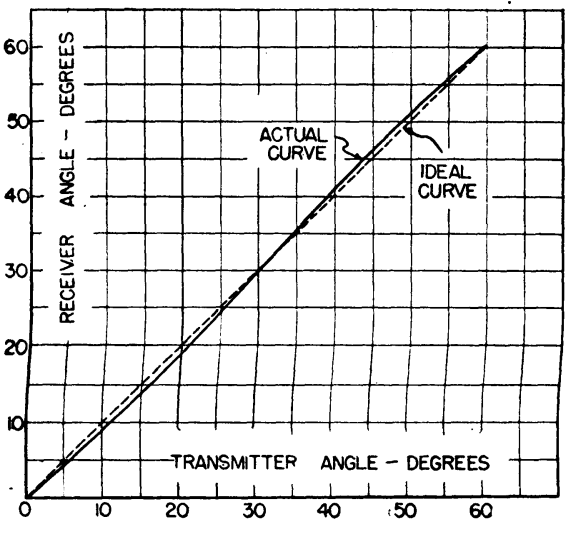

Figure 6. Variation of receiver angle with transmitter angle

to zero, we obtain the value of $\theta$ for which $\beta$ is a maximum. ${ }^{*}$ This gives values of $\theta=13$ degrees 18 minutes and 46 degrees 42 minutes. The corresponding maximum values of $\beta$ are 1 degree 6 minutes and -1 degree 6 minutes, respectively.

It is also of interest from a design standpoint to investigate the variation of the magnitude of the resultant field as $\theta$ is varied, since the friction error will depend on this magnitude. To do this we investigate first the case for $r \rightarrow \infty$, that is when the receiver resistance is many times larger than the transmitter resistance. Then $\left(-\theta^{2}+60 \theta+1,800\right)$ is negli= gible compared to $5,400 \mathrm{r}$ in equations 7 , 8 , and 9 . These equations become

$I_{C A}=\frac{E}{180 R_{2}}$

$I_{A B}=\frac{\cdot E(60-\theta)}{10,800 R_{2}}$

$I_{B C}=\frac{-E \theta}{10,800 R_{2}}$

Figure 8 shows the current distribution in the coils for $\theta$ from 0 to 360 degrees. Equations 7, 8, and 9 are valid only from 0 to 60 degrees, but the rest of the cycle can readily be seen to be as shown. Substituting the foregoing values in equations 10 and 11 , we obtain

$\phi_{x}=K\left(2-\frac{\theta}{60}\right)$.

$\phi_{y}=K \sqrt{3} \frac{\theta}{60}$

where $K=$ constant.

These are the parametric equations of one side of a regular hexagon centered at the origin and having a vertex on the $x$ axis. The plot is shown in Figure 9. The line $A B C$ is the locus of the ends of the flux vector as $\theta$ varies from 0 to $6 \theta$ degrees.

To obtain the locus of the end of the flux vector for any value of $r$, we simply note that all the currents given by equa-

* See Appendix I

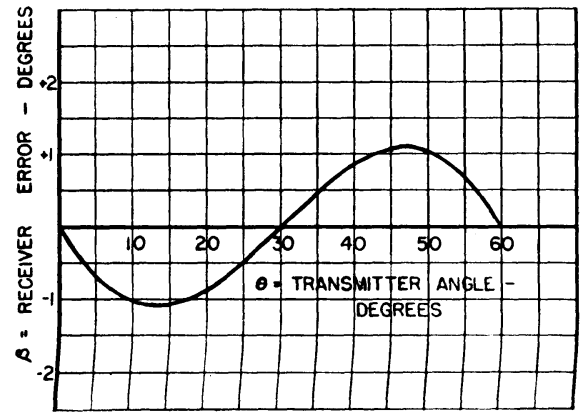

Figure 7. Natural error in Selsyn receiver

$$
\beta=\tan ^{-1}\left(\frac{\sqrt{ } \overline{3} \theta}{120-\theta}\right)-\theta
$$

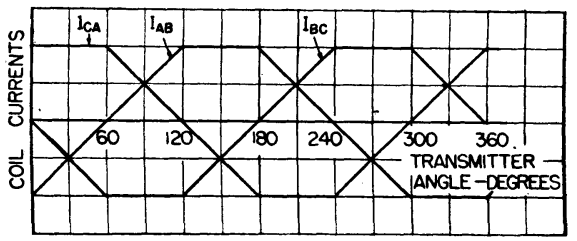

Figure 8. Current distribution in receiver coils, $r=R_{2} / R_{1}$

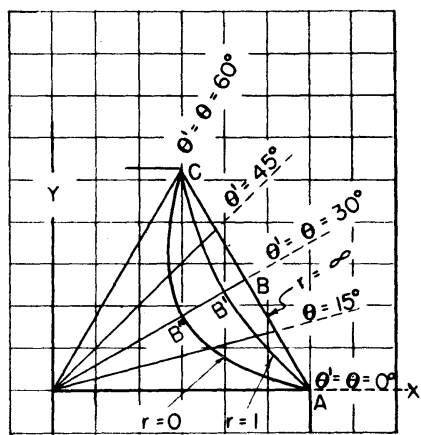

Figure 9. Loci of ends of flux vector for various resistance ratios

tions $7^{\prime}, 8^{\prime}$, and $9^{\prime}$ will be equal to the currents given by equations 7,8 , and 9 when the former are multiplied by a factor proportional to $1 /\left(-\theta^{2}+60 \theta+1,800+\right.$ $5,400 r)$. Hence, the vectors ending on the line $A B C$ need only be multiplied by the afore-mentioned factor to give the locus for any value of $r$. It is to be noted that the angle the flux vector makes with the $x$ axis is $\theta^{\prime}$, not $\theta$, and hence equations $10^{\prime}$ and $11^{\prime}$ multiplied by the foregoing factor should be used to determine the locus. The lines $A B^{\prime} C$ and $A B^{\prime \prime} C$ show the locus for $r=1$ and $r=0$, respectively. For comparison purposes, the maximum values in each case have been made to coincide. The actual value of maximum flux depends on $R_{1}$ and $R_{2}$, but this is discussed in the second part of the report. Figure 10 shows the variation of current in each coil for $r=1$.

It may be seen from Figure 9 that the flux reaches a minimum every 60-degree interval of transmitter rotation. In the analysis which follows, design considerations will be based on making this mini-

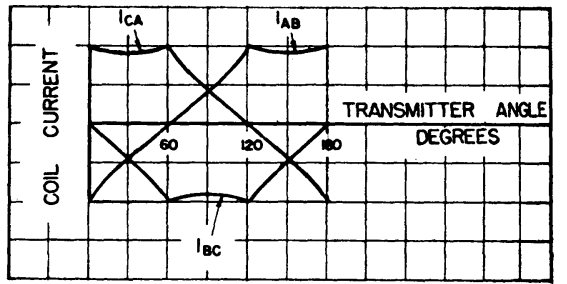

Figure 10. Current distribution in receiver, coils for $R_{2} / R_{1}=1$

mum value of flux as large as possible for given power. As we shall see, this is not the same as making the maximum flux as large as possible.

\section{Optimum Transmitter and Receiver Resistances}

For a given amount of power, and a given amount of space in which to wind the receiver coil, it is possible to determine optimum resistances for both transmitter and receiver and hence the wire size to be used in the receiver. It is also possible to determine the best size of receiver coil, consistent with space limitations.

Let us assume that the coil is to have the dimensions shown in Figure 11.

\section{$l=$ length of one turn \\ $a=$ cross-section area of coil \\ $\mathrm{N}$ = number turns on one coil \\ $A=$ cross-section area of wire}

We then have

$120 R_{2}=$ total resistance of coil $=\frac{\rho 1 N}{A}$

$N A=\alpha a$

where $\alpha$ is a spacing factor.

$\alpha=0.91$

We wish to make the resultant flux a maximum when $\theta=30$ degrees, which is the weak flux point. This amounts to making $N I$ in the coil $C A$ a maximum for $\theta=30$ degrees. Using equation 7 , we have

$$
\begin{aligned}
N I & =\frac{30 E}{R_{1}} \frac{N}{2,700+5,400 r} \\
& =\frac{E}{96} \frac{N}{R_{1}+2 R_{2}}
\end{aligned}
$$

Using equations 15 and 16 , we eliminate $N$ and obtain

$N I=\mid K \frac{R_{2^{1} / 2}}{R_{1}+2 R_{2}}$

where

$K=\frac{E}{45} \sqrt{\frac{30 \alpha a}{\rho 1}}$

It is evident from equation 18 that $K$ is made large by increasing $a$, decreasing $\rho$, or decreasing $l$ for a given supply 


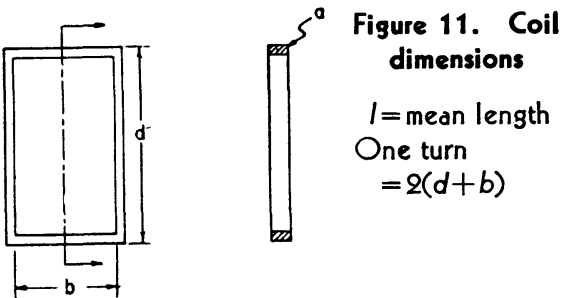

voltage. It is not advisable to decrease $l$, since the enclosed area of the coil is proportional to torque for given $N I$. However, for best results, $a$ should be made as large as possible and $\rho$ as low as possible, that is, low-resistivity material should be used.

The maximum power consumed in the circuit is given by

$P=\frac{E^{2}}{R_{\min }}$

where $R_{\min }$ is the least value of resistance between battery terminals. From Figure 4 we see that

$R_{\min }=\frac{30 R_{1}}{R_{1}+R_{2}}\left(R_{1}+3 R_{2}\right)$

and since

$P=\frac{E^{2}}{30} \frac{R_{1}+R_{2}}{R_{1}\left(R_{1}+3 R_{2}\right)}$

from which

$R_{2}=\frac{R_{1}\left(1-\frac{30 P}{E^{2}} R_{1}\right)}{\left(\frac{90 P}{E^{2}} R_{1}-1\right)}$

Substituting value of $R_{2}$ from 20 in equation 18 , we obtain

$N I=K \frac{\left(-3 K^{\prime 2} R_{1}^{2}+4 K^{\prime} R_{1}-1\right)^{1 / 2}}{R_{1}^{1 / 2}\left(K^{\prime} R_{1}+1\right)} ; K^{\prime}=\frac{30 P}{E^{2}}$

By differentiating expression 21 with respect to $R_{1}$ and equating result to zero, we obtain, for maximum $N I^{*}$

$R_{1}=\frac{1+2 \sqrt{2}}{7 K^{\prime}}=0.547 \frac{E^{2}}{30 P}$

$120 R_{1}=$ resistance per leg $=2.19 \frac{E^{2}}{P}$

and from equation 20

$R_{2}=R_{1} \frac{(3-\sqrt{2})}{(3 \sqrt{2}-2)}=\frac{1}{\sqrt{2}} R_{1}$

With the optimum values determined from equations 22 and 23 , the size wire for the receiver may be calculated from equations 15 and 16.

Small variations of the receiver resistance from the optimum value will not affect the performance appreciably, although power consumption, of course,

\footnotetext{
* See Appendix II.
}

will change. This may be seen by writing equation 18 in the form:

$N I=\frac{K}{R_{1}^{1 / 2}} \frac{r^{1 / 2}}{(1+2 r)}$

Equation 18 is plotted in Figure 12 for both $R_{1}$ constant and $R_{2}$ constant. It may be seen that the variation of $N I$ with $R_{2}$ is small, whereas the variation with $R_{1}$ is greater, in the region of $r=\frac{1}{\sqrt{2}}$.

Design equations based on making the flux a maximum in the strongest point, that is, when $\theta=0$, lead to somewhat different equations. . Analysis similar to the foregoing yields the following results:

$120 R=$ resistance per leg $=2.30 \frac{E^{2}}{P}$

$R_{2}=\frac{R_{1}}{\sqrt{3}}$

For best performance, equations 22 and 23 , not $22^{\prime}$ and $23^{\prime}$, should be used.

\section{Correction of Natural Error in Selsyn System}

In the first part of this report it was found that the error is zero in the reeeiver when the transmitter angle $\theta$ (see Figure 1) is $0,30,60$ degrees, and so forth. Since one of the brushes is in contact with a terminal every 60 degrees, it is obvious that any nonlinear resistance must be symmetrical on either side of every 0 . 60,120 degrees, and so forth. Furthermore, since the natural error for a linear resistance is symmetrical and of opposite sign on either side of every 30 -degree point, we may assume that the nonlinear resistance should be symmetrical on either side of $0,30,60$ degrees, and so on.

The foregoing considerations reduce the problem to one of finding the deviation from linearity for the region from 0 to 30 degrees, making the deviation from 30 to 60 degrees symmetrical about the 30-degree point and of opposite sign, then repeating the cycle every 60 degrees.

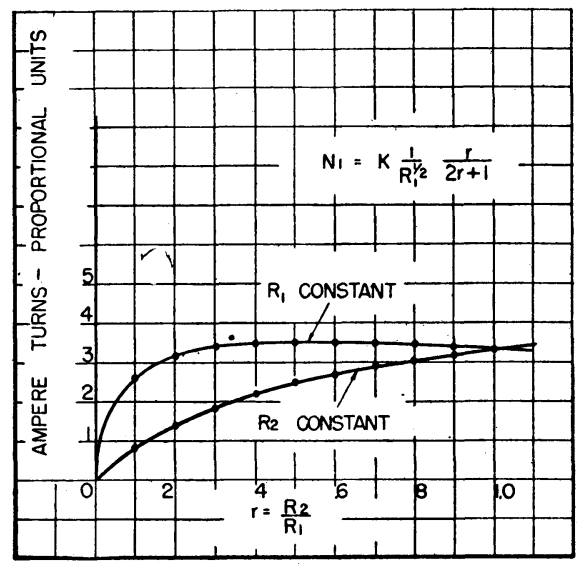

Figure 12. Variation of ampere turns with $R_{1}$ and $R_{2}$ constant power
Referring to Figure 1, we let the resistance of each transmitter leg be $120 R_{1}$, and the resistance of each receiver leg $120 R_{2}$, as before. For $\theta \leqslant 30$ degrees, let the resistance of that portion of the winding marked (1) be equal to $\alpha R_{1}$. The problem now resolves itself into one of finding $\alpha$ as a function of $\theta$ such that the exror shall be zero for all values of $\theta$ from zero to 30 degrees.

Remembering the symmetry which we have noted, the values of the resistances (1) to (5) of the transmitter then must be

(1) $=\alpha R_{1}$

(2) $=(120-\alpha) R_{1}$

(3) $=(60+\alpha) R_{1}$

(4) $=(60-\alpha) R_{1}$

(5) $=120 R_{1}$

Referring to Figure 2, we see that the afore-mentioned resistances are exactly the same as those shown in Figure 2, with $\alpha$ substituted for $\theta$. In our present situation, then, it is obvious that the receiver angle $\theta^{\prime}$, will be given by equation 13, with $\alpha$ substituted for $\theta$, or

$\tan \theta^{\prime}=\frac{\sqrt{3} \bar{\alpha}}{120-\alpha}$

In prder to have zero error, $\theta=\theta^{\prime}$, and $\tan \theta=\sqrt{3} \alpha /(120-\alpha)$.

Solving for $\alpha$, we obtain

$\alpha=\frac{120 \tan \theta}{\sqrt{3}+\tan \theta}$

This gives the necessary variation of $\alpha$ with $\theta$ for zero error and hence the variation of resistance with $\theta$, since the resistance of segment (1) is $\alpha R_{1}$.

Figure 13 shows the variation of $\alpha$ with $\theta$, along with the linear variation, so that the deviation may be noted.

It is to be especially noted that the variation of $\alpha$ with $\theta$ for zero error is independent of the receiver resistance and hence will work for all receivers or paralleled receivers.

To obtain the shape of the developed cylinder which will give a resistance in accordance with equation 25 , we refer to Figure 14. $O A B C$ represents 30 degrees

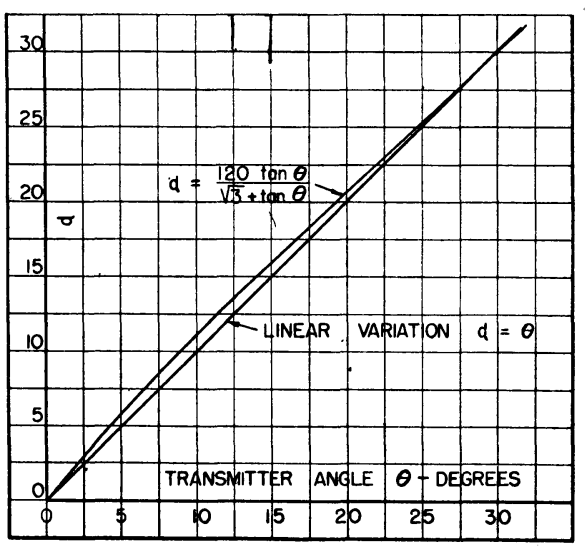

Figure 13. Variation of modification factor with transmitter angle $\theta$ 


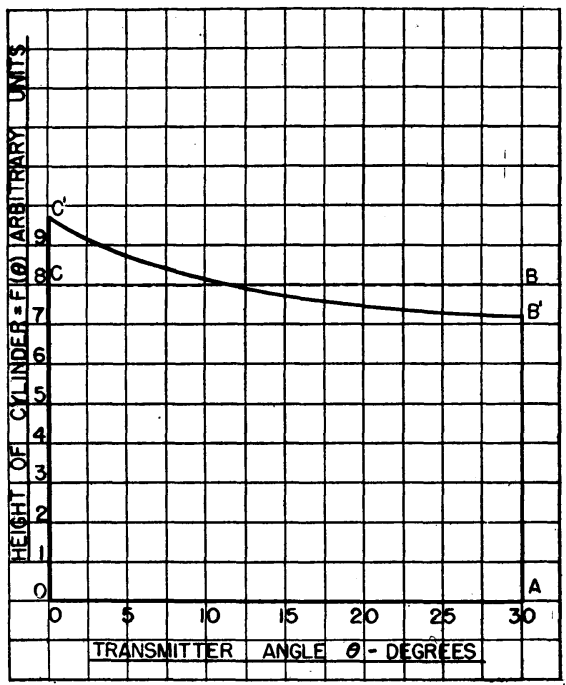

Figure 14. $\boldsymbol{F}(\theta)$ for 0 degrees $\leqq \theta \leqq 30$ degrees

of the developed cylinder for a linear resistor. $O A B^{\prime} C^{\prime}$ represents the shape of 30 degrees of the developed cylinder for the nonlinear resistor.

Let us denote the equation of $C^{\prime} B^{\prime}$ as $F(\theta)$. For a particular value of $\theta$, the ratio of the nonlinear resistance to the linear resistance will be equal to the ratio of the area beneath the curve $C^{\prime} B^{\prime}$ to the area beneath the curve $C B$. This ratio is obtained from the equations of the two curves in Figure 13. Hence we have

$$
\begin{aligned}
\frac{\int_{0}^{\theta} F(\theta) d \theta}{L \theta} & =\frac{120 \tan \theta}{\sqrt{3}+\tan \theta} \cdot \frac{\pi}{180 \theta} \\
& =\frac{2 \pi}{3 \theta} \frac{\tan \theta}{\sqrt{3}+\tan \theta}
\end{aligned}
$$

where $\theta$ is expressed in radians, and $L=O C$ in Figure 15.

If we solve for the numerator in the lefthand side,

$$
\int_{0}^{\theta} F(\theta) d \theta=\frac{2 \pi \hbar}{3} \frac{\tan \theta}{\sqrt{3}+\tan \theta}
$$

If we differentiate both sides with respect to $\theta$,

$$
\begin{aligned}
F(\theta) & =\frac{2 \pi L}{3} \frac{d}{d \theta}\left(\frac{\tan \theta}{\sqrt{3}+\tan \theta}\right) \\
& =\frac{2 \pi L}{3} \frac{1}{(\sqrt{3} \cos \theta+\sin \theta)^{2}}
\end{aligned}
$$

Figure 15 shows $F(\theta)$ plotted against $\theta$ from 0 to 30 degrees. $O A B^{\prime} C^{\prime}$ is thus the developed shape of 30 degrees of the toroidal resistor. The developed shape for the whole resistor is shown in Figure 15.

\section{Conclusions}

In a d-c Selsyn a natural-error cycle exists which has a 60 -degree period. The error is positive for 30 degrees and negative for 30 degrees, reaching a maximum of 1 degree 6 minutes at 13 degrees 18 minutes and 46 degrees 42 minutes on the transmitter.

Design equations to give maximum flux in receiver for a given voltage and power are given by

Transmitter resistance $=2.19 \frac{E^{2}}{P}$ ohms per leg $=R_{T}$

Receiver resistance $=\frac{1}{\sqrt{2}} R_{T}$ ohms per leg

Furthermore, the cross-sectional area of the receiver coil should be as large as

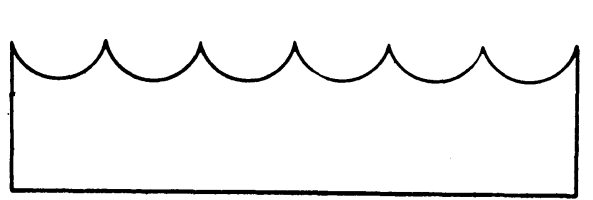

Figure 15. Developed shape of cylinder on which transimitter-resistor may be wound in order to produce zero error

is practical; and the resistivity of the receiver coil as low as possible.

The material and design dimensions of the transmitter coil are immaterial insofar as flux properties are concerned.

The natural error of a d-c Selsyn system may be eliminated completely by winding the transmitter resistance in a nonlinear manner.

The necessary variation of resistance with transmitter angle to give zero error is independent of receiver resistance. This correction for natural error may be attained for all possible loads on the transmitter.

One method of obtaining the desired resistor is to wind it on a cylinder whose developed shape is shown in Figure 16. This is by no means the only way of accomplishing the desired result. Any method which will make the resistance vary in accordance with equation 25 is satisfactory from an operational viewpoint. From a manufacturing viewpoint there may be more desirable methods than that of modifying the shape of the toroid on which the resistor is wound.
Appendix I. Maximum Natural Error in Selsyn

If we use equation 14, and express angles in radians, so that we may differentiate

$$
\beta=\frac{\theta}{57.3}-\tan ^{-1} \frac{\sqrt{3} \theta}{120-\theta}
$$

$\frac{d \beta}{d \theta}=\frac{1}{57.3}-\frac{1}{1+\left(\frac{\sqrt{3} \theta}{120-\theta}\right)^{2}} \times$

$$
\begin{gathered}
\frac{(120-\theta) \sqrt{3}+\sqrt{3} \theta}{(120-\theta)^{2}} \\
=\frac{1}{57.3}-\frac{120 \sqrt{3}}{\left(120^{2}-240 \theta+4 \theta^{2}\right)}=0
\end{gathered}
$$

for maximum $\beta$

If we solve,

$\left(\frac{\theta}{60}\right)^{2}+\left(\frac{\theta}{60}\right)+1=\frac{57.3 \sqrt{ } \overrightarrow{3}}{120}=0.828$

$\left(\frac{\theta}{60}\right)^{2}+\left(\frac{\theta}{60}\right)+0.172=0 ;$

$\frac{\theta}{60}=\frac{1 \pm \sqrt{1-0.69}}{2}=0.778$ or 0.222

$\theta=13.3^{\circ}, 46.7^{\circ}$

\section{Appendix II. Conditions for Maximum $\mathrm{NI}$ in Receiver}

If we use equation 21 ,

$N I=K \frac{\Delta}{R_{1}{ }^{1 / 2}\left(K^{\prime} R_{1}+1\right)}$

where

$$
\Delta=\left[-3 K^{\prime 2} R_{1}{ }^{2}+4 K^{\prime} R_{1}-1\right]^{1 / 2}
$$

$\frac{d(N I)}{d R_{1}}=0$

$$
=K\left[\begin{array}{c}
R_{1}{ }^{1 / 2}\left(K^{\prime} R_{1}+1\right) \cdot 1 / 2 \Delta^{-1} \times \\
\left(-6 K^{\prime 2} R_{1}+4 K^{\prime}\right)- \\
\frac{\Delta\left\{R_{1}{ }^{1 / 2} K^{\prime}+R_{1}{ }^{-1 / 2}\left(K^{\prime} R_{1}+1\right)\right\}}{R_{1}\left(K^{\prime} R_{1}+1\right)^{2}}
\end{array}\right]
$$

Setting numerator equal to zero, we have

$R_{1}\left(K^{\prime} R_{1}+1\right)\left(-6 K^{\prime 2} R_{1}+4 K^{\prime}\right)=$ $\left(-3 K^{\prime 2} R_{1}{ }^{2}+4 K^{\prime} R_{1}-1\right)\left(R_{1} K^{\prime}+K^{\prime} R_{1}+1\right)$

If we multiply and separate powers of $R_{\mathrm{l}}$,

$-7 K^{2} R_{1}{ }^{2}+2 K^{\prime} R_{1}+1=0$

$R_{1}=\frac{1}{K^{\prime}} \frac{-2 \pm \sqrt{4+28}}{14}=\frac{1}{K^{\prime}} \frac{1 \pm \sqrt{8}}{7}$

If we discard negative values of $R_{1}$ as meaningless.

$R_{1}=\frac{1+2 \sqrt{2}}{7 K^{\prime}}=\frac{0.547}{K^{\prime}}$ 